\title{
Comparison of Intravenous and Non-Intravenous Antibiotic Regimens in Eradication of P. aeruginosa and MRSA in Cystic
}

\section{Fibrosis}

Huseyin Hakan Mursaloglu ${ }^{1}$, Can Akin ${ }^{2}$, Cansu Yilmaz Yegit ${ }^{3}$, Almala Ergenekon ${ }^{2}$, Burcu Uzunoglu $^{2}$, Gamze Tastan ${ }^{2}$, Yasemin Gokdemir ${ }^{3}$, Ela Erdem Eralp ${ }^{4}$, Aysegul Karahasan ${ }^{2}$, Fazilet Karakoc ${ }^{4}$, and Bulent Karadag ${ }^{4}$

${ }^{1}$ Marmara University Training and Research Hospital

${ }^{2}$ Marmara University School of Medicine

${ }^{3}$ Marmara University

${ }^{4}$ Marmara University Faculty of Medicine

May 6, 2021

\begin{abstract}
Background: Chronic pulmonary infection is the leading cause of mortality and morbidity in patients with cystic fibrosis (CF). The most common pathogens isolated in CF are Staphylococcus aureus and Pseudomonas aeruginosa (P. aeruginosa). Chronic infection of P. aeruginosa and MRSA are associated with worse survival and antibiotic eradication treatment is recommended for both. This study evaluates the efficacy of intravenous (IV) vs. non-IV antibiotics in the eradication of P. aeruginosa and MRSA. Methods: This was a single-center retrospective study at a large CF center. All respiratory specimen cultures of 309 CF patients and eradication regimens between 2015-2019 were reviewed. The primary analysis was the comparison of the percentage of successful eradication after receiving IV and non-IV eradication regimens. Demographic and clinical risk factors for eradication failure were also analyzed. Results: 102 patients with P. aeruginosa isolations and 48 patients with MRSA were analyzed. At one year, $21.6 \%$ in P. aeruginosa group and 35.4\% in MRSA group were successfully eradicated. There was not any statistically significant difference between IV vs. non-IV antibiotic regimens on eradication in either group. Additionally, none of the clinical risk factors was significantly associated with eradication failure in P. aeruginosa and MRSA groups. Conclusion: In the eradication of P. aeruginosa and MRSA, IV and non-IV treatment regimens did not show any superiority to one another. Non-parenteral eradication could be a better option in eradication considering the cost-effectiveness and the treatment burden of IV treatments due to hospitalization and the need for IV access.
\end{abstract}

\section{Comparison of Intravenous and Non-Intravenous Antibiotic Regimens in Eradication of $\boldsymbol{P}$. aeruginosa and MRSA in Cystic Fibrosis}

\section{Authors:}

H. Hakan Mursaloğlu ${ }^{1}$ (e-mail: hhakanmursaloglu@gmail.com)

Can $\mathrm{Akın}^{2}$ (e-mail: cancecigerce@gmail.com)

Cansu Yılmaz Yeğit ${ }^{3}$, (e-mail: cansuuuuyilmaz@gmail.com)

Almala P. Ergenekon ${ }^{3}$, (e-mail: drpergenekon@hotmail.com)

Burcu Suzer Uzunogluํㅜ, (e-mail: burcusuzer@gmail.com)

Gamze Taştan ${ }^{1}$, (e-mail: gamzetastan977@hotmail.com) 
Yasemin Gökdemir ${ }^{3}$, (e-mail: yasemingokdemir@yahoo.com.tr)

Ela Erdem Eralp ${ }^{3}$, (e-mail: elaerdem@yahoo.com)

Ayşegül Karahasan Yağcl${ }^{4}$, (e-mail: aysegulkarahasan@gmail.com)

Fazilet Karakoç3 ${ }^{3}$ (e-mail: infofaziletkarakoc@gmail.com)

Bulent Karadağ ${ }^{3}$ (e-mail: bkaradag@hotmail.com)

\section{Affiliations:}

${ }^{1}$ Department of Pediatric Pulmonology; Selim Coremen Cystic Fibrosis Center, Marmara University Faculty of Medicine, Istanbul, Turkey.

${ }^{2}$ Faculty of Medicine, Marmara University, Istanbul, Turkey

${ }^{3}$ Department of Pediatric Pulmonology, Faculty of Medicine, Marmara University, Istanbul, Turkey

${ }^{4}$ Medical Microbiology Department, Marmara University Faculty of Medicine, Istanbul

\section{Corresponding Author:}

Dr. Hüseyin Hakan Mursaloğlu

Address: Suadiye Mh. Sebboy Sk. No:2 D:16, 34740, Istanbul/Turkey

Tel: +905364132020

e-Mail: hhakanmursaloglu@gmail.com

\section{Abstract}

Background: Chronic pulmonary infection is the leading cause of mortality and morbidity in patients with cystic fibrosis (CF). The most common pathogens isolated in CF are Staphylococcus aureus and Pseudomonas aeruginosa ( $P$. aeruginosa). Chronic infection of $P$. aeruginosa and MRSA are associated with worse survival and antibiotic eradication treatment is recommended for both. This study evaluates the efficacy of intravenous (IV) vs. non-IV antibiotics in the eradication of $P$. aeruginosa and MRSA.

Methods: This was a single-center retrospective study at a large CF center. All respiratory specimen cultures of 309 CF patients and eradication regimens between 2015-2019 were reviewed. The primary analysis was the comparison of the percentage of successful eradication after receiving IV and non-IV eradication regimens. Demographic and clinical risk factors for eradication failure were also analyzed.

Results: 102 patients with $P$. aeruginosa isolations and 48 patients with MRSA were analyzed. At one year, $21.6 \%$ in $P$. aeruginosa group and $35.4 \%$ in MRSA group were successfully eradicated. There was not any statistically significant difference between IV vs. non-IV antibiotic regimens on eradication in either group. Additionally, none of the clinical risk factors was significantly associated with eradication failure in P. aeruginosa and MRSA groups.

Conclusion: In the eradication of $P$. aeruginosa and MRSA, IV and non-IV treatment regimens did not show any superiority to one another. Non-parenteral eradication could be a better option in eradication considering the cost-effectiveness and the treatment burden of IV treatments due to hospitalization and the need for IV access.

Keywords: Cystic fibrosis, Pseudomonas Aeruginosa, MRSA, eradication, antibiotic, chronic infection

\section{Abbreviations}

CF: Cystic Fibrosis

CFTR: Cystic fibrosis transmembrane conductance regulator 
TORPEDO-CF: Trial of Optimal Therapy for Pseudomonas Eradication in Cystic Fibrosis

\section{Introduction}

Cystic fibrosis $(\mathrm{CF})$ is the most common life-limiting autosomal recessive disease in Caucasian populations. Thickened mucous secretion caused by the mutations on the CFTR gene leads to obstruction in the bronchioles secondary to mucus plugging. This causes an optimal environment for bacterial growth and infection ${ }^{1}$. Chronic pulmonary infection damaging the lung parenchyma and decreasing the pulmonary function is the leading cause of mortality and morbidity in individuals with $\mathrm{CF}^{2-4}$. The most common microorganisms isolated from respiratory secretions in $\mathrm{CF}$ areStaphylococcus aureus (S. aureus) and Pseudomonas aeruginosa (P. aeruginosa $)^{4}$.

Chronic infection of $P$. aeruginosa is associated with more parenchymal damage, a more rapid decline in lung function, and earlier mortality ${ }^{2 ; 5}$. The acquisition ofMethicillin-resistant S. aureus (MRSA), even though that is not seen as much as $P$. aeruginosa, causes a decline in forced expiratory volume in 1-second percent predicted $\left(\mathrm{FEV}_{\mathrm{pp}}\right)$ and associated with worse survival ${ }^{6 ; 7}$. Current guidelines recommend antibiotic eradication treatment for MRSA and P. aeruginosa to prevent lung damage and chronic infections $8 ; 9$.

The antibiotic eradication treatment for $P$. aeruginosa and MRSA is an established standard of care worldwide $^{9-13}$. However, the most effective route of administration of antibiotics (oral, inhalation or intravenous (IV)) and choice of antibiotic regimen are not clear. The two Cochrane reviews on this matter state that there is still insufficient data to decide on which antibiotic strategy should be used for the eradication of early $P$. aeruginosa or MRSA in $\mathrm{CF}^{14 ; 15}$.

There are numerous randomized controlled trials (RCT) on comparing different oral antibiotic regimens, oral vs. inhalation regimens and oral/inhalation combination regimens for P. aeruginosaeradication ${ }^{14}$. While there are many retrospective studies comparing inhalation and oral antibiotic regimens, there are only two RCTs for MRSA eradication ${ }^{15}$. On the other hand, there are few studies comparing IV vs. oral/inhalation antibiotic regimens. To date, there is only one RCT comparing IV and non-parenteral antibiotics for the eradication of $P$. aeruginosa and there have been no published data that investigate the efficacy of IV compared to non-parenteral antibiotics to eradicate MRSA ${ }^{16}$.

Today, many CF clinics use IV antibiotics as antibiotic eradication treatment. IV eradication regimen requires hospital admission, IV access which might be traumatic and may worsen patient's health-related quality of life (HRQoL) ${ }^{17}$. Additionally, IV eradication regimens increase health-care costs significantly ${ }^{16}$. In this study, we investigate the efficacy of IV vs. non-IV (oral and/or inhalation) antibiotic eradication treatments for the eradication of the first isolation of P. aeruginosa and MRSA in individuals with CF. Additionally, we aim to determine clinical variables associated with chronic infection of $P$. aeruginosa and MRSA.

\section{Materials and Methods}

This was a single-center retrospective study conducted at the Marmara University Medical Faculty Selim Çöremen CF Center between January 2015 and December 2019. 309 patients with CF were involved in the study. The inclusion criteria were: CF diagnosis according to the consensus guidelines from the Cystic Fibrosis Foundation ${ }^{18}$, regular follow-up in the CF center outpatient clinic and having at least 4 sputum or deep pharyngeal swab cultures per year (every 2-4 months).

All sputum and deep pharyngeal swab cultures collected from subjects between 2015-2019 were reviewed. Patients who had their first isolation of $P$. aeruginosa or MRSA were grouped separately. Patients' demographics, anthropometric measurements, clinical findings, chest physiotherapy (CPT) techniques, regular treatments and eradication regimens were obtained from the medical records.

Chronic or intermittent infection of $P$. aeruginosa or MRSA were determined according to the Leeds Criteria $^{19}$. The Leeds Criteria defines chronic infection as having positive P. aeruginosa or MRSA in more than $50 \%$ of the cultures in the preceding year and intermittent infection as having positive P. aeruginosa 
or MRSA in less than 50\%. Successful eradication was set as not having any isolation of P. aeruginosa or MRSA during 12 months after having the eradication regimen for their first isolation. Patients were followed up at least one year after their first isolation of $P$. aeruginosa or MRSA.

The primary analysis of the study was the comparison of the percentage of successful eradication after receiving IV and non-IV (oral and/or inhalation) eradication regimens. Sex, diagnosis age, age at isolation, body mass index (BMI) z-score, [?]F508 mutation, pancreatic insufficiency, CF-related Diabetes Mellitus (CFRD), biliary-hepatic disease, azithromycin use, dornase-alfa use, inhaled corticosteroid use, allergic bronchopulmonary aspergillosis (ABPA), previousmethicillin-sensitive S. aureus (MSSA), chest physiotherapy and FEV1pp were analyzed as predictors of eradication failure.

The aim of the study was explained and informed consent was obtained from the participants beforehand. The Marmara University Medical Faculty Research Ethics Committee had approved the study with protocol number 09.2020.509.

Hospital eradication protocol

All patients with the first isolation of $P$. aeruginosa and MRSA were offered IV antibiotic eradication treatment. 14 days IV ceftazidime and amikacin were used for $P$. aeruginosa eradication. For the eradication of MRSA, 14 days IV teicoplanin was used in addition to 5 days $2 \%$ nasal mupirocin ointment and 5 days $4 \%$ chlorhexidine body wash. The regimens were adapted if a patient had any allergies or was resistant to any of the first-line antibiotics. If a patient did not accept hospitalization, an oral and/or inhalation eradication regimen was chosen by specialist decision.

Collection and processing of Respiratory Tract Samples

Respiratory samples taken from CF patients including sputum and deep pharyngeal swab samples (DPS) collected with ESwab (Copan Diagnostics, Murrieta, CA) sent to the microbiology laboratory for processing and inoculation. An equal volume of Sputasol solution (Thermo Scientific, USA) was added to the sputum samples and homogenized in a shaker incubator for 1 hour at $37 \mathrm{degC}$. The samples were vortexed in every 15 minutes and the resulting homogenates were inoculated whereas DPS samples were processed directly. Samples were inoculated into Mac Conkey agar, $5 \%$ sheep-blood agar, Haemophilus selective agar (chocolate agar including bacitracin), Burkholderia cepacia selective agar (BCSA), CHROMID ${ }^{(\mathrm{r})}$, S. aureus Elite agar (SAIDE) agar plates then incubated for 24-72 hours at $35.5 \mathrm{degC}$. All plates were purchased from bioMerieux. Culture media evaluated daily for significant bacterial growth and if colonies exist, identification of microorganisms done by Matrix-Assisted Laser Desorption/Ionization time-of-flight, Mass Spectrometry (MALDI-TOF MS, BioMerieux, France). Antimicrobial susceptibility testing has done by Kirby-Bauer disk diffusion method and Vitek 2 system (bioMerieux). Results were evaluated according to the European Committee on Antimicrobial Susceptibility Testing (EUCAST).

\section{Statistical Analysis}

Continuous variables were presented as mean +- standard deviation (SD). Baseline patient characteristics and study outcomes were analyzed between IV and non-IV treatment arms in P. aeruginosa and MRSA groups separately. Continuous data were analyzed via independent-samples $\mathrm{T}$ test or Mann-Whitney U tests for non-parametric data. Categorical data were analyzed via $\chi 2$ or Fisher's exact tests. P-value [?] 0.05 was considered to be significant. Statistical analysis was performed using the IBM Statistical Package for the Social Sciences (SPSS) Version 23.

\section{Results}

Demographics and medical data

A total of $309 \mathrm{CF}$ patients were involved in the study. Of these 309 patients, 120 had their first isolation of $P$. aeruginosa and 61 had first isolation of MRSA during the study period (2015-2019). 18 patients from $P$. aeruginosa group and 13 patients from MRSA group were excluded from the study due to lack of medical records or delayed treatment due to the presentation to the clinic later than the first month of isolation. 
Patients' baseline characteristics are presented in Table 1. Baseline characteristics were similar between IV and non-IV treatment arms except patients who had IV treatment in $P$. aeruginosa group were younger (aged $5.1 \pm 4.9$ vs $8.1 \pm 5.8 \mathrm{p}=0.02$ ).

Pseudomonas Aeruginosa Group (N=102)

The mean age of the patients at first isolation of $P$. aeruginosawas $5.83 \pm 5.28$ years. The mean duration from diagnosis to first $P$. aeruginosa isolation was $4.16 \pm 4.0$ years. While 22 isolations $(21.6 \%)$ from patients were successfully eradicated at 1 year after their first isolation, $56(54.9 \%)$ had intermittent and $24(23.5 \%)$ had chronic infection (Table 2).

The most common preferred eradication regimen in our clinic was intravenous amikacin and ceftazidime for 14 days. Table 2 shows the successful eradication rates by antibiotic regimens. The highest success rate among treatment regimens was 14 days ciprofloxacin PO +28 days inhaled tobramycin by $62.5 \%$ vs. IV amikacin + ceftazidime by $20.3 \%$ but this difference was not statistically significant $(\mathrm{p}>0.05)$. When IV amikacin + ceftazidime and other non-intravenous treatment regimens compared, there was not any statistically significant difference $(\mathrm{p}=0.54)$. Results of antibiotic susceptibility testing showed $89.2 \%$ of isolations were sensitive to all antibiotics and $10.8 \%$ were resistant to one or more antibiotics. Patients received their eradication regimens according to the antibiotic susceptibility of their microbial cultures.

Sex, diagnosis age, age at isolation, BMI z-score, $\Delta$ F508 mutation, pancreatic insufficiency, CFRD, biliaryhepatic disease, dornase alfa use, inhaled corticosteroid use, ABPA, hospitalization in the preceding year and $\mathrm{FEV}_{1 \mathrm{pp}}$ were not statistically significant as a predictor $(\mathrm{p}>0.05)$.

26 out of 102 patients were able to perform spirometry. The mean FEV1pp of eradicated group $(\mathrm{n}=6)$ was $81.6 \pm 9.02$ and not eradicated group $(\mathrm{n}=20)$ was $91.8 \pm 18.65$. This difference was not statistically significant $(\mathrm{p}>0.05)$.

Methicillin-Resistant Staphylococcus Aureus Group ( $\mathrm{N}=48$ )

The mean age of the patients at first isolation of MRSA was $7.66 \pm 5.71$ years. The mean duration from diagnosis to first MRSA isolation was $6.3 \pm 5.25$ years. While 17 isolations $(35.4 \%)$ were successfully eradicated at 1 year, 17 patients $(35.4 \%)$ had intermittent and 14 (29.2\%) had chronic infection (Table 2).

The most common MRSA eradication regimen used in our clinic was intravenous teicoplanin for 14 days. The highest success rate among treatments was 14 days oral Trimethoprim / Sulfamethoxazole (TMP/SMX) + Rifampicin by $50 \%$ eradication success. Statistical analysis did not show any difference between treatment regimens $(\mathrm{p}>0.05)$. Additionally, successful eradication rates were not significantly different between intravenous and oral treatment groups $(\mathrm{p}=0.57)$.

Sex, diagnosis age, age at isolation, BMI z-score, $\Delta$ F508 mutation, pancreatic insufficiency, CFRD, biliaryhepatic disease, dornase alfa use, inhaled corticosteroid use, ABPA, hospitalization rate in the preceding year and FEV1pp were not statistically significant as a predictor $(\mathrm{p}>0.05)$.

20 out of 48 patients were able to perform spirometry. The mean FEV1pp of patients in eradicated and not eradicated groups were $88.9 \pm 21$ and $93.2 \pm 13.1$, respectively $(\mathrm{p}>0.05)$.

\section{Discussion}

The present study was designed to evaluate the efficacy of IV antibiotics compared to non-IV eradication regimens. IV antibiotic treatment is considered to be more effective compared to other routes in pulmonary exacerbation and it is recommended for the patients with moderate to severe pulmonary exacerbation or with no response to oral and/or inhalation antibiotics ${ }^{20}$. There is a limited data on IV vs oral and/or inhalation antibiotic regimens for the eradication of early P. aeruginosa or MRSA acquisition. The data from our study demonstrated that there is not any statistically significant difference between IV vs non-IV (oral and/or inhalation) antibiotic eradication regimens in terms of eradication at one year of the first isolation of $P$. aeruginosa and MRSA. 
IV treatment often requires hospitalization, might have a negative effect on HRQoL in individuals with CF and costs significantly more than oral/inhalation treatments ${ }^{16 ; 21}$. Although a number of studies have been reported the effectiveness of oral, inhalation and IV eradication protocols, there are only a few comparative studies available and the optimal antibiotic regimens for both MRSA and P. aeruginosa are not clear. $12 ; 15 ; 22$.

A recent $\mathrm{RCT}$ on $P$. aeruginosa eradication compared the efficacy of 14 days IV ceftazidime and tobramycin vs 12 weeks oral ciprofloxacin; both groups were combined with 12 weeks inhaled colistimethate sodium ${ }^{16}$. In line with our results, IV antibiotics did not achieve sustained eradication of $P$. aeruginosa in a greater proportion of patients with CF compared to the oral therapy group. The study also evaluated the cost-effectiveness and found that oral eradication therapy has similar effectiveness for the primary clinical parameters and considerably cheaper compared to IV treatment.

Even though non-parenteral eradication protocols have been more commonly used since they have similar effectiveness with IV treatment and more preferable method in terms of cost effectiveness and patients QoL, insufficient effect of inhaled antibiotics on inflammation is a valid concern. A RCT comparing the systemic antibiotics to inhaled tobramycin alone in terms of reduction in lower airways inflammation suggests that systemic antibiotic treatment has a greater effect on reducing lower airways inflammation, even though systemic and inhaled treatments appeared to have similar effects on reduction of bacterial burden ${ }^{23}$. These results might cause concerns about the impact of inflammation in lower airways such as increase in bronchiectasis and decrease in pulmonary function. However, studies did not show any difference in FEV1 $1_{\text {pp }}$ change between inhaled with oral antibiotics and only inhaled antibiotic regimens ${ }^{24 ; 25}$.

There are several MRSA eradication protocols evaluated in the literature including very mild regimens such as single or dual oral antibiotics and very aggressive treatments such as dual iv antibiotic treatment over 3 weeks followed by a 6 -week period with dual oral antibiotic therapy and inhalation with vancomycin, all in addition to environmental hygienic directives and topical therapies ${ }^{22 ;}$ 26-31. These studies have been reported successful eradication rates from $41.4 \%$ to $84 \%$ with the first isolation for MRSA. The successful eradication rate of MRSA in our study cohort was $35.4 \%$. Our eradication rate was lower than other studies and this might be due to the different study end-points. We determined the successful eradication as not having any isolations 12 months after the treatment compared to evaluating at 28-day of the treatment in most of the studies in the literature.

To the best of our knowledge, this is the first study comparing IV and non-IV antibiotics for the eradication of MRSA. Our results indicate that oral antibiotics (TMP/SMX with or without rifampicin) may be as efficient as IV teicoplanin for the eradication treatment.

Determining risk factors influencing acquisition and eradication of MRSA and P. aeruginosa in CF is crucial for prevention and treatment success. To date, studies showed lower socio-economic status, female gender, $\Delta$ F508 mutation and pancreatic insufficiency as risk factors for $P$. aeruginosa ${ }^{32 ;} 33$. Additionally, retrospective cohort studies of individuals with CF in the Cystic Fibrosis Foundation Patient Registry demonstrated that pancreatic insufficiency, CFRD and number of hospitalizations in the preceding year were the most common risk factors for eradication failure of $\mathrm{MRSA}^{34 ; 35}$. In our study, none of these clinical risk factors were significantly associated with eradication failure both in P. aeruginosa and MRSA groups.

Our study has a number of limitations. Firstly, this is a retrospective design and the generalizability of the results is limited due to being a single-center study. Also, while our sample size is relatively bigger than comparative studies in the literature, most patients were given IV treatment due to the hospital protocol and this limited the numbers of non-IV treatment cohort.

In conclusion, we did not find any difference between IV and non-IV treatments in successful eradication at 1 year for both $P$. aeruginosa and MRSA. This study, in line with TORPEDO-CF, shows that IV treatment does not add any benefit in the eradication of $P$. aeruginosa ${ }^{16}$. Additionally, eradication rates were not significantly different in the eradication of MRSA and this should be verified by further prospective studies. In light of these results, cost-effectiveness and to decrease the treatment burden due to hospitalization and 
IV administration of antibiotics, we have increased the use non-parenteral eradication protocols in our CF clinic.

\section{Acknowledgements}

This research did not receive any specific grant from funding agencies in the public, commercial, or not-forprofit sectors.

\section{Conflict of interest}

None declared.

1. Yu E, Sharma S. 2020. Cystic fibrosis. Statpearls. Treasure Island (FL): StatPearls Publishing

Copyright (C) 2020, StatPearls Publishing LLC.

2. Rosenfeld M, Gibson RL, McNamara S, Emerson J, Burns JL, Castile R, Hiatt P, McCoy K, Wilson CB, Inglis A et al. 2001. Early pulmonary infection, inflammation, and clinical outcomes in infants with cystic fibrosis. Pediatr Pulmonol. 32(5):356-366.

3. Zolin A OA, Naehrlich L, Jung A, van Rens J et al. 2020. Ecfspr annual report 2018.

4. Cystic fibrosis foundation patient registry, 2018 annual data report, bethesda, maryland.

5. Emerson J, Rosenfeld M, McNamara S, Ramsey B, Gibson RL. 2002. Pseudomonas aeruginosa and other predictors of mortality and morbidity in young children with cystic fibrosis. Pediatr Pulmonol. 34(2):91-100.

6. Vanderhelst E, De Meirleir L, Verbanck S, Piérard D, Vincken W, Malfroot A. 2012. Prevalence and impact on fev1 decline of chronic methicillin-resistant staphylococcus aureus (mrsa) colonization in patients with cystic fibrosis: A single-center, case control study of 165 patients. Journal of Cystic Fibrosis. 11(1):2-7.

7. Dasenbrook EC, Checkley W, Merlo CA, Konstan MW, Lechtzin N, Boyle MP. 2010. Association between respiratory tract methicillin-resistant staphylococcus aureus and survival in cystic fibrosis. JAMA. 303(23):2386-2392.

8. Cystic fibrosis trust. Recommendations - eradication and treatment of mrsa. In: Antibiotic treatment for cystic

fibrosis. 3rd edn. Bromley, cystic fibrosis trust, 2009.

9. Mogayzel PJ, Jr., Naureckas ET, Robinson KA, Brady C, Guill M, Lahiri T, Lubsch L, Matsui J, Oermann CM, Ratjen F et al. 2014. Cystic fibrosis foundation pulmonary guideline. Pharmacologic approaches to prevention and eradication of initial pseudomonas aeruginosa infection. Ann Am Thorac Soc. 11(10):16401650.

10. Chmiel JF, Aksamit TR, Chotirmall SH, Dasenbrook EC, Elborn JS, LiPuma JJ, Ranganathan SC, Waters VJ, Ratjen FA. 2014. Antibiotic management of lung infections in cystic fibrosis. I. The microbiome, methicillin-resistant staphylococcus aureus, gram-negative bacteria, and multiple infections. Ann Am Thorac Soc. 11(7):1120-1129.

11. Taccetti G, Bianchini E, Cariani L, Buzzetti R, Costantini D, Trevisan F, Zavataro L, Campana S, Italian Group for PaEiCF. 2012. Early antibiotic treatment for pseudomonas aeruginosa eradication in patients with cystic fibrosis: A randomised multicentre study comparing two different protocols. Thorax. 67(10):853-859.

12. Döring G, Flume P, Heijerman H, Elborn JS. 2012. Treatment of lung infection in patients with cystic fibrosis: Current and future strategies. J Cyst Fibros. 11(6):461-479.

13. Palser S, Smith S, Nash EF, Agarwal A, Smyth AR. 2019. Treatments for preventing recurrence of infection with pseudomonas aeruginosa in people with cystic fibrosis. Cochrane Database Syst Rev. 12:CD012300. 
14. Langton Hewer SC, Smyth AR. 2017. Antibiotic strategies for eradicating pseudomonas aeruginosa in people with cystic fibrosis. Cochrane Database of Systematic Reviews. (4).

15. Lo DK, Muhlebach MS, Smyth AR. 2018. Interventions for the eradication of meticillin-resistant staphylococcus aureus (mrsa) in people with cystic fibrosis. Cochrane Database Syst Rev. 7:CD009650.

16. Hewer SCL, Smyth AR, Brown M, Jones AP, Hickey H, Kenna D, Ashby D, Thompson A, Williamson PR. 2020. Intravenous versus oral antibiotics for eradication of pseudomonas aeruginosa in cystic fibrosis (torpedo-cf): A randomised controlled trial. Lancet Respir Med. 8(10):975-986.

17. Yohannes AM, Willgoss TG, Fatoye FA, Dip MD, Webb K. 2012. Relationship between anxiety, depression, and quality of life in adult patients with cystic fibrosis. Respir Care. 57(4):550-556.

18. Farrell PM, White TB, Ren CL, Hempstead SE, Accurso F, Derichs N, Howenstine M, McColley SA, Rock M, Rosenfeld M et al. 2017. Diagnosis of cystic fibrosis: Consensus guidelines from the cystic fibrosis foundation. The Journal of Pediatrics. 181:S4-S15.e11.

19. Lee TWR, Brownlee KG, Conway SP, Denton M, Littlewood JM. 2003. Evaluation of a new definition for chronic pseudomonas aeruginosa infection in cystic fibrosis patients. Journal of Cystic Fibrosis. 2(1):29-34.

20. Doring G, Conway SP, Heijerman HG, Hodson ME, Hoiby N, Smyth A, Touw DJ. 2000. Antibiotic therapy against pseudomonas aeruginosa in cystic fibrosis: A european consensus. Eur Respir J. 16(4):749-767.

21. Vandekerckhove K, Keyzer M, Cornette J, Coomans I, Pyl F, De Baets F, Schelstraete P, Haerynck F, De Wolf D, Van Daele S et al. 2017. Exercise performance and quality of life in children with cystic fibrosis and mildly impaired lung function: Relation with antibiotic treatments and hospitalization. Eur J Pediatr. 176(12):1689-1696.

22. Akil N, Muhlebach MS. 2018. Biology and management of methicillin resistant staphylococcus aureus in cystic fibrosis. Pediatr Pulmonol. 53(S3):S64-S74.

23. Noah TL, Ivins SS, Abode KA, Stewart PW, Michelson PH, Harris WT, Henry MM, Leigh MW. 2010. Inhaled versus systemic antibiotics and airway inflammation in children with cystic fibrosis and pseudomonas. Pediatr Pulmonol. 45(3):281-290.

24. Treggiari MM, Retsch-Bogart G, Mayer-Hamblett N, Khan U, Kulich M, Kronmal R, Williams J, Hiatt P, Gibson RL, Spencer T et al. 2011. Comparative efficacy and safety of 4 randomized regimens to treat early pseudomonas aeruginosa infection in children with cystic fibrosis. Archives of Pediatrics \& Adolescent Medicine. 165(9):847-856.

25. Proesmans M, Vermeulen F, Boulanger L, Verhaegen J, De Boeck K. 2013. Comparison of two treatment regimens for eradication of pseudomonas aeruginosa infection in children with cystic fibrosis. J Cyst Fibros. 12(1):29-34.

26. Poster session abstracts. 2016. Pediatric Pulmonology. 51(S45):S194-S485.

27. Belarski E, Pettit R. 2020. Outcomes of a methicillin-resistant staphylococcus aureus (mrsa) eradication protocol in pediatric cystic fibrosis (cf) patients. Pediatr Pulmonol. 55(3):654-659.

28. Doe SJ, McSorley A, Isalska B, Kearns AM, Bright-Thomas R, Brennan AL, Webb AK, Jones AM. 2010. Patient segregation and aggressive antibiotic eradication therapy can control methicillin-resistant staphylococcus aureus at large cystic fibrosis centres. J Cyst Fibros. 9(2):104-109.

29. Dolce D, Neri S, Grisotto L, Campana S, Ravenni N, Miselli F, Camera E, Zavataro L, Braggion C, Fiscarelli EV et al. 2019. Methicillin-resistant staphylococcus aureus eradication in cystic fibrosis patients: A randomized multicenter study. PLOS ONE. 14(3):e0213497.

30. Kappler M, Nagel F, Feilcke M, Kroner C, Pawlita I, Naehrig S, Ripper J, Hengst M, von Both U, Forstner M et al. 2016. Eradication of methicillin resistant staphylococcus aureus detected for the first time 
in cystic fibrosis: A single center observational study. Pediatr Pulmonol. 51(10):1010-1019.

31. Vallieres E, Rendall JC, Moore JE, McCaughan J, Hoeritzauer AI, Tunney MM, Elborn JS, Downey DG. 2016. Mrsa eradication of newly acquired lower respiratory tract infection in cystic fibrosis. ERJ Open Res. 2(1).

32. Jackson L, Waters V. 2021. Factors influencing the acquisition and eradication of early $<\mathrm{em}>$ pseudomonas aeruginosa $</$ em $>$ infection in cystic fibrosis. Journal of Cystic Fibrosis. 20(1):8-16.

33. Rosenfeld M, Emerson J, McNamara S, Thompson V, Ramsey BW, Morgan W, Gibson RL. 2012. Risk factors for age at initial $<\mathrm{em}>$ pseudomonas $</ \mathrm{em}>$ acquisition in the cystic fibrosis epic observational cohort. Journal of Cystic Fibrosis. 11(5):446-453.

34. Dasenbrook EC, Merlo CA, Diener-West M, Lechtzin N, Boyle MP. 2008. Persistent methicillin-resistant staphylococcus aureus and rate of fev1 decline in cystic fibrosis. Am J Respir Crit Care Med. 178(8):814-821.

35. Jennings MT, Dasenbrook EC, Lechtzin N, Boyle MP, Merlo CA. 2017. Risk factors for persistent methicillin-resistant staphylococcus aureus infection in cystic fibrosis. J Cyst Fibros. 16(6):681-686.

\section{Hosted file}

Table 1.pdf available at https://authorea.com/users/361347/articles/521001-comparison-ofintravenous-and-non-intravenous-antibiotic-regimens-in-eradication-of-p-aeruginosa-andmrsa-in-cystic-fibrosis

\section{Hosted file}

Table 2.pdf available at https://authorea.com/users/361347/articles/521001-comparison-ofintravenous-and-non-intravenous-antibiotic-regimens-in-eradication-of-p-aeruginosa-andmrsa-in-cystic-fibrosis

\section{Hosted file}

Table 3.pdf available at https://authorea.com/users/361347/articles/521001-comparison-ofintravenous-and-non-intravenous-antibiotic-regimens-in-eradication-of-p-aeruginosa-andmrsa-in-cystic-fibrosis

\section{Hosted file}

Table 4.pdf available at https://authorea.com/users/361347/articles/521001-comparison-ofintravenous-and-non-intravenous-antibiotic-regimens-in-eradication-of-p-aeruginosa-andmrsa-in-cystic-fibrosis 